\title{
Stall Recovery of a Morphing Wing via Extended Nonlinear Lifting-Line Theory
}

\author{
Lawren L. Gamble* \\ University of Michigan, Ann Arbor, Michigan 48109 \\ Alexander M. Pankonien \pm \\ U.S. Air Force Research Laboratory, Dayton, Ohio 45433 \\ and \\ Daniel J. Inman \\ University of Michigan, Ann Arbor, Michigan 48109 \\ DOI: $10.2514 / 1 . J 055042$
}

\begin{abstract}
Aircraft morphing provides advantages to traditional flight including drag reduction and maneuverability. Previous research indicates that smooth spanwise transitions in trailing-edge camber, representative of a biological analog, provide aerodynamic benefits at small angles of attack by eliminating vortices at geometric discontinuities but lack nonlinear aerodynamic investigations. This work aims to analyze the adaptability of a spanwise morphing wing concept with respect to nonlinear aerodynamics using an optimized nonlinear extended lifting-line model. In this novel approach, it is shown that adaptation, including stall recovery, can be achieved solely through geometric tailoring as opposed to attitude correction for a range of flight conditions while reducing the drag penalty associated with operating at the unadapted condition. The range of conditions for which the wing can recover are restricted by the limited trailing-edge deflections and the inability of the actuators to substantially shift the stall angle of the section lift curve. These results provide insight into improving morphing wing designs, indicating that, by adding another degree of freedom to the chordwise deformation such as a morphing hinge capable of larger actuation and reflex camber, stall recovery via geometric tailoring may be feasible for an even larger range of conditions.
\end{abstract}

\section{Nomenclature}

$A=$ aspect ratio

$C_{D}=$ wing drag coefficient

$C_{D, i}=$ induced wing drag coefficient

$C_{L}=$ wing lift coefficient

$C_{l}=$ section lift coefficient

$c=$ chord

$d C_{l}=$ change in section lift coefficient

$d \alpha_{\text {eff }}=$ change in effective angle of attack

$j=$ spanwise section number

$n=$ iteration number

$w_{\text {ind }}=$ induced downwash

$y_{j}=$ spanwise section location

$\alpha_{\text {eff }}=$ effective angle of attack

$\alpha_{\text {geo }}=$ geometric angle of attack

$\alpha_{0}=$ zero-lift angle of attack

$\Gamma=$ circulation distribution

$\delta_{\text {tip }}=$ trailing-edge linear deflection

$\eta=$ trailing-edge angular deflection

$\eta_{k}=$ spanwise integration points

$\mu \quad=$ artificial viscosity term

$\omega=$ relaxation factor

\section{Introduction}

A KEY advantage of morphing unmanned aerial vehicles (UAVs) over their rigid and discrete counterparts is their ability to adapt to multiple flight conditions via geometric tailoring as birds do in nature. Birds capabilities to morph their wings provide a highly effective method of adapting to adverse aerodynamic conditions due to wind gusts, prey payload, etc. [1]. As an example, the steppe eagle is capable of deliberately stalling sections of their wings [2], motivating research into spanwise morphing wing studies. This ability to morph geometry allows both birds and aircraft to operate effectively over a range of flight conditions. In aircraft, this allows for efficient flight outside of the principal design by altering parameters such as camber, sweep, twist, or planform area [3] ]. In small UAVs that operate at low Reynolds numbers, this can be advantageous because unfavorable aerodynamic phenomenon such as wind gusts and static imbalance due to payload shift and delivery can heavily impact gliding flight. Yet, although research within aircraft morphing has been rapidly growing in recent years, the focus has lingered on linear aerodynamic conditions.

Tremendous advances and novel morphing ideas have recently been developed for operation at cruise conditions. At transonic speeds, such advances include NASA's Variable Camber Continuous Trailing Edge Flap (VCCTEF) [4-6] and the FlexSys Mission Adaptive Compliant Wing (MACW) [7]. The VCCTEF harnessed the rapid time-scale benefits of conventional electromechanical motors in addition to the weight-to-power benefits of shape memory alloy torque tubes. Alternately, the MACW used conventional actuators for adequate power consumption, bandwidth, and range of displacement to tailor the airfoil camber of an optimized internal compliant structure which maintained reasonable stresses, stability, dynamic behavior, etc. These advances highlight the importance of developing integrated morphing technologies for commercial and military aircraft operating at cruising speeds because even slight decreases in drag can amount to tremendous savings in fuel consumption. However, with the simultaneous advancement of smart materials, three-dimensional (3-D) printed compliant skins, and sensor networks, new morphing concepts on the UAV scale have been enabled. publication 12 March 2017; published online 25 May 2017. Copyright (C) 2017 by the authors. Published by the American Institute of Aeronautics and Astronautics, Inc., with permission. All requests for copying and permission to reprint should be submitted to CCC at www.copyright.com; employ the ISSN 0001-1452 (print) or 1533-385X (online) to initiate your request. See also AIAA Rights and Permissions www.aiaa.org/randp.

*Ph.D. Candidate, Department of Aerospace Engineering, 1320 Beal Avenue. Student Member AIAA.

${ }^{\dagger}$ National Research Council Postdoctoral Researcher, Aerospace Systems Directorate, 1864 4th Street. Member AIAA.

${ }^{\ddagger}$ Chair, Department of Aerospace Engineering, 1320 Beal Avenue. Fellow AIAA. 
By introducing one or more of these advancements into a single design, morphing UAVs are capable of outperforming their traditional counterparts. Such morphing concepts include the cascading bimorph concept developed by Bilgen et al. []ㅡ, which achieved a camber morphing airfoil using macrofiber composite (MFC) actuators, and the spanwise morphing trailing edge (SMTE) concept developed by Pankonien and Inman [9-11], which was designed to achieve continuous shape change across six spanwise piezoelectric actuators. Pankonien and Inman's work, directly relevant to the current study, optimized the spanwise deflections of a morphing finite wing using Prandtl's lifting-line theory (LLT) to recover from an off-design flight condition at small angles of attack and showed that the SMTE, which exhibits smooth spanwise changes in trailing-edge deflection, provided substantial drag reduction due to the elimination of vortex formations at surface discontinuities when compared to a wing with rigid discrete control surfaces of equivalent spanwise geometry. However, the assessment of aerodynamic performance of morphing aircraft at large angles and near stall has been limited in part by difficulties in modeling the poststall aerodynamic behavior of a finite wing.

Accordingly, this paper builds upon the work conducted by Pankonien and Inman and investigates the capabilities of the SMTE to adapt to nonlinear aerodynamics, which is then applied to stall recovery, by tailoring the trailing-edge deflection of the six actuators. To predict the actuator configurations for stall recovery, a model that accurately captures the nonlinear behavior of finite wing aerodynamics must be used. The most widely used nonlinear LLT models are frequently criticized for their inability to converge past stall (where the lift-curve slope is negative [12]), sawtooth oscillations in their poststall circulation distributions, and nonunique poststall solutions [13], making them unideal for poststall analysis and optimization. A more recent nonlinear method developed by Chattot resolved these issues by incorporating an artificial viscosity term into the governing equation and consequently showed excellent poststall agreement with an analytical solution [14].

Using this loosely coupled viscous model, this work aims to quantify the capabilities of a morphing wing to adapt to nonlinear aerodynamics and recover from stall. The scenario under investigation assumes that an aircraft adapts to unfavorable aerodynamic conditions, detailed as follows [11]. The aircraft begins at its designed cruise conditions (on-design). Following a change in aerodynamic load such as a gust of wind or payload delivery, which may change the location of the center of gravity, the aircraft now operates at an off-design condition defined by a change in angle of attack. This paper will focus on the analysis of off-design conditions near stall where such changes could have disastrous consequences due to the severe decrease in lift and increase in drag. While remaining at the off-design state, the aircraft adapts to the unfavorable aerodynamics by morphing the spanwise trailing-edge deflections to return to the initial on-design lift condition. Although this final flight condition will incur a drag penalty with respect to the on-design flight condition, this penalty will be substantially lower than it would be in the absence of adaptation, as can be seen in Fig. 1. Overall, this proposed method of adaptation differs from the traditional reduced angle approach to stall recovery due to differences in the aerodynamic conditions between transonic aircraft and small UAVs. Furthermore, it is important to note that, although the motivation for this work is partly attributed to wind gusts, the focus is on prolonged disturbances like payload delivery. Transient loadings are not considered in this initial analysis; thus, a steady aerodynamic model is used.

This adaptation scenario is specific to nontraditional aircraft including but not limited to low-speed aircraft or small UAVs, which operate in urban environments and gustier conditions, and variablesweep wings, which are prone to changes in stall location. This proposed spanwise variation in trailing-edge camber allows the actuators to target specific stalled regions of the wing such as the root or tip, inspired by the adaptation of the steppe eagle. This ability would prove particularly useful for variable-sweep wings where the location of stall can vary between the inboard and outboard wing, depending on the sweep configuration. Although the model proposed here is not valid for largely swept wings, this does not detract from the SMTE's ability to tailor the spanwise geometry in the aforementioned scenario. Furthermore, with the MFC's rapid actuation time, the spanwise shape of the wing can be quickly tailored to mitigate the effects of the nonlinear aerodynamics while in the disturbed state. Then, when the disturbance has passed, the wing will return to its original on-designed geometry. The alternative for traditional aircraft would be to either wait until the disturbance has passed, incurring an unadapted drag penalty shown in Fig. 1 by the difference between points 2 and 1, or change the angle of attack via elevator control, which would need to be restored after the disturbance had passed, resulting in a sinusoidal pitching motion and possible instabilities. Furthermore, extended disturbances such as payload deliveries will change the total lift and may alter the location of the center of gravity, resulting in long-term change in angle of attack. Like birds, which change their wing geometry while carrying prey, the wing design considered here is capable of morphing the spanwise shape to adapt to this change in payload. Finally, with the ongoing development of superior sensor networks and control laws that can respond semi-instantaneously, the change in angle between the initial and disturbed states would be small. Thus, it is clear to imagine how this method of adaptation can be used to reject disturbances immediately, allowing for more extreme maneuvers, near-stall flight, and more stable longitudinal flight, which (as an example) would be largely beneficial in flying wings with limited control surfaces or UAVs carrying fixed cameras.

Previous research by Gamble et al. has shown through the proposed model and accompanying experiments that this method of recovery is successful in both a spanwise morphing wing and a discrete flap wing of equal spanwise capabilities and has highlighted the superior performance of the morphing wing [15]. The current work builds upon this knowledge to quantify the limitations of such extreme adaptation with respect to the initial on-design lift constraint $\left(\mathrm{C}_{L, \text { on-design }}\right)$ and the off-design angle of attack $\left(\alpha_{\text {off-design }}\right)$. This was accomplished by optimizing the actuator deflections and thus the spanwise geometry of the wing, using the viscous nonlinear liftingline model to return to the on-design lift condition using the geometry of the SMTE. Overall, the following work serves to develop a model capable of analyzing the nonlinear aerodynamics of a spanwise morphing wing, analyze the extent of adaptable scenarios including

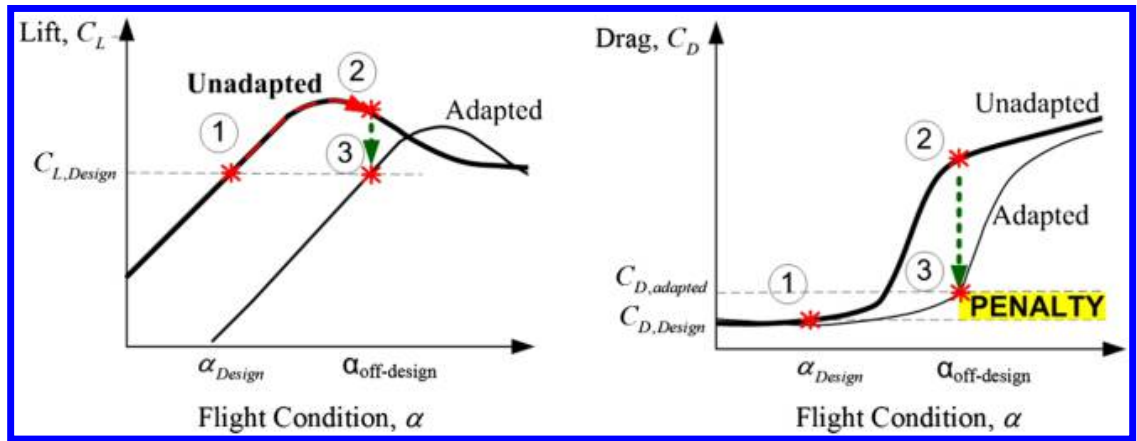

Fig. 1 Graphic summary of the design scenario in terms of forces, depicting the adapted aerodynamic curves and associated drag penalty (between dashed lines). 
stall recovery, contribute to the understanding of the strengths of spanwise morphing aircraft when handling nonlinear aerodynamics, and inform future morphing designs by suggesting critical improvements.

\section{Nonlinear Lifting-Line Theory Model}

To identify and quantify nonlinear aerodynamic adaptation in a spanwise morphing aircraft, an analytical model was required to predict the aerodynamic behavior of a finite wing in both the linear and nonlinear regions of the lift curve. As justified in the prior section, Chattot's viscous nonlinear model was chosen. Upon validation of the model with experimental results, the model was used as the foundation for a constrained optimization that tailored the spanwise deflection of the wing to return to the on-design lift condition at the off-design angle of attack. The wing successfully recovered if it was capable of matching the on-design lift while at the off-design angle; however, this method can be extended to stall recovery as well. The morphing wing was said to have successfully recovered from stall if, after adaptation, the off-design angle of the new wing configuration was less than or equal to the angle of maximum lift of the unadapted wing. This was computed at a variety of on- and off-design conditions to quantify the limitations of nonlinear adaptation and stall recovery for a spanwise morphing aircraft.

\section{A. Static Two-Dimensional Simulations}

Two-dimensional static simulations were generated using OVERTURNS [16,17], a Reynolds-averaged Navier-Stokes solver for three airfoil shapes and angles of attack ranging from -25 to $25 \mathrm{deg}$ in increments of $1 \mathrm{deg}$. A symmetric NACA 0012 airfoil was chosen for the spanwise unactuated geometry. The geometry for the actuated (i.e., morphed, adapted) airfoil was chosen to have the same geometry as the SMTE, which will be discussed in the latter section, to include the conformal bending produced by the macrofiber composite (MFC) actuators. This geometry can be seen in Fig. 2. This was used to obtain the two-dimensional (2-D) section lift, profile drag, and pitching moment curves, which will be used in the nonlinear lifting-line model.

\section{B. Nonlinear Lifting-Line Model}

The loosely coupled circulation based method uses Prandtl's dimensionless integrodifferential equation to model the 3-D effects of a finite wing and is written as

$$
\Gamma(y)=\frac{1}{2} c(y) C_{l}\left[\alpha_{\text {geo }}-\alpha_{0}(y)+\arctan \left(w_{\text {ind }}(y)\right)\right]
$$

where the circulation has been normalized by the freestream velocity. The wing is first discretized into spanwise stations with a cosine distribution generating a higher resolution at the tips to better capture the effects of the trailing vortices for $j=1,2, \ldots, j x$, where

$$
y_{j}=-\cos \left(\frac{j-1}{j x-1} \pi\right)
$$

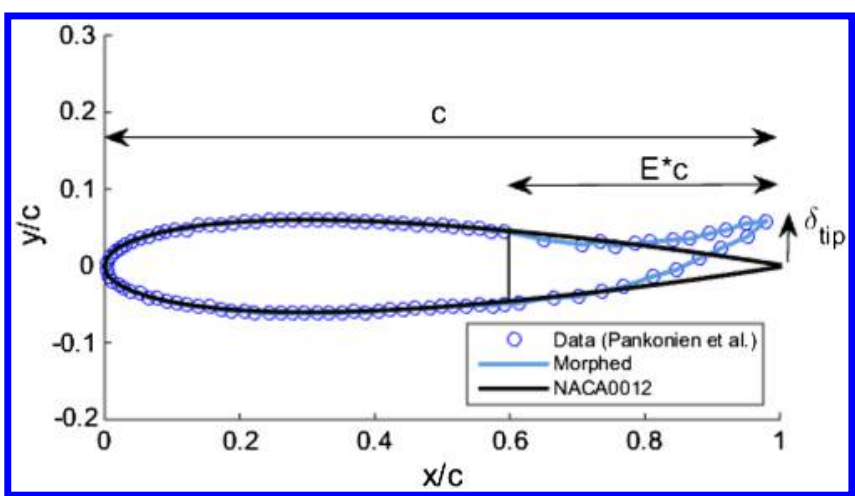

Fig. 2 Morphed geometry of the airfoil profile compared to the unactuated geometry [18]. and for this particular analysis, $j x$ was 49 , comparable with previous studies. Integration points are defined between each of the stations to avoid singularities when calculating the induced downwash for $k=1,2, \ldots, j x-1$, where

$$
\eta_{k}=-\cos \left(\frac{k-(1 / 2)}{j x-1} \pi\right)
$$

Then, an initial circulation distribution, often elliptical, is assumed to seed the model with a solution, after which the induced downwash at each section can be calculated using a finite sum:

$$
w_{\text {ind }, j}=-\frac{1}{4 \pi} \sum_{k=1}^{j x-1} \frac{\Gamma_{k+1}^{n}-\Gamma_{k}^{n}}{y_{j}-\eta_{k}}
$$

Next, Prandtl's integrodifferential governing equation is linearized using Newton's method given by

$$
\Gamma_{j}+\Delta \Gamma_{j}=\frac{1}{2} c_{j}\left(C_{l, j}+\frac{d C_{l, j}}{d \alpha_{\mathrm{eff}}} \Delta \alpha_{\mathrm{eff}, j}\right)
$$

where

and

$$
\Delta \alpha_{\mathrm{eff}, j}=\frac{\Delta w_{\mathrm{ind}, j}}{1+w_{\mathrm{ind}, j}^{2}}
$$

$$
\Delta w_{\text {ind }, j}=-\frac{1}{4 \pi}\left(\frac{1}{y_{j}-\eta_{j-1}}-\frac{1}{y_{j}-\eta_{j}}\right) \Delta \Gamma_{j}=a_{j} \Delta \Gamma_{j}
$$

This can then be written in iterative form, and the artificial viscosity term can be added to both the right and left hand sides of the governing equation, so that the spanwise stations are now coupled, resulting in

$$
\begin{aligned}
(1 & \left.-\frac{1}{2} c_{j} \frac{d C_{l, j}}{d \alpha_{\mathrm{eff}}} \frac{a_{j}}{1+w_{\mathrm{ind}, j}^{2}}+2 \mu\right) \frac{\Delta \Gamma_{j}}{\omega} \\
= & \frac{1}{2} c_{j} C_{l, j}-\Gamma_{j}^{n}+\mu\left(\Gamma_{j+1}^{n}-2 \Gamma_{j}^{n}+\Gamma_{j-1}^{n+1}\right)
\end{aligned}
$$

where

$$
\left\{\begin{array}{c}
\mu \geq \max \left(\frac{1}{4} c_{j} \frac{d C_{l, j}}{d \alpha_{\mathrm{eff}}} \frac{a_{j}}{\left(1+w_{\mathrm{ind}, j}^{2}\right)} ; 0\right) \\
\omega<1 \text { (under relaxed) }
\end{array}\right.
$$

where a relaxation factor of 0.8 was used in this analysis. This value was chosen because iterative stability is guaranteed when $\omega<1$, but the model further approximates the solution as the relaxation factor decreases. By rewriting the iterative circulation distribution as $\Gamma_{j-1}^{n+1}=\Gamma_{j-1}^{n}+\Delta \Gamma_{j-1}$ and substituting this into Eq. (ㅁ), the linearized integrodifferential equation is reduced to one unknown $\Delta \Gamma_{j}$. This is then used to calculate the next circulation distribution $\Gamma_{j}^{n+1}=\Gamma_{j}^{n}+\Delta \Gamma_{j}$. Equations (4-6) are then reiterated until the solution converges to the desired convergence criteria. For this analysis, a strict convergence criteria of $0.01 \%$ was chosen, where the circulation at every spanwise station must satisfy the criteria. Upon convergence, the lift and drag of the finite wing can be calculated using the converged circulation solution as

$$
\begin{gathered}
C_{L}=\frac{\Re}{2} \int_{-1}^{1} \Gamma(y) \mathrm{d} y \\
C_{D, \text { Total }}=C_{D, \text { profile }}+\overbrace{\frac{R}{-2} \int_{-1}^{1} \Gamma(y) w(y) \mathrm{d} y}^{C_{D, i}}
\end{gathered}
$$

where the integrals have been written using substitution so that the bounds of integration are normalized. As is, this model works well for 


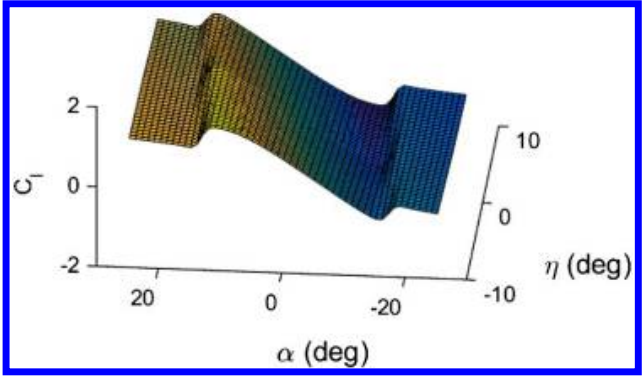

Fig. 3 Three-dimensional surface of section lift data with respect to angle of attack and trailing-edge deflection.

predicting the aerodynamic performance of static wing shapes such as wing/winglet combinations, turbine blades, and swept wings shown by Chattot $[19,20]$ and Gallay et al. [21]. However, the section-based approach is ideal for analyzing morphing wings that are not constrained to a given spanwise geometry.

\section{Morphing Geometry Implementation}

In spanwise morphing wings, the section lift and its respective derivative are no longer simply a function of the angle of attack; rather, the trailing-edge deflection adds a second parameter that must also be considered, where

$$
\eta=\tan ^{-1}\left(\frac{\delta_{\text {tip }}}{E^{*} c}\right)
$$

Accordingly, both the trailing-edge deflection and angle of attack are required to determine the lift coefficient at each section. Thus, the model requires a bounded domain of airfoil characteristics to interpolate from. This domain can be generated in the form of a surface, shown in Fig. 3, which correlates the lift coefficient to both the angle of attack and trailing-edge deflection. From observations of the simulated data, the section lift curves vary linearly with respect to the trailing-edge deflection; thus, linearly interpolating the curves from simulated airfoil data at the maximum, minimum, and neutral trailing-edge deflections is appropriate. Thus, by knowing the spanwise shape function of the morphing wing's trailing edge for arbitrary actuator displacements, the section lift characteristics at and between the actuators can be found.

This shape function, which describes the spanwise variation in trailing-edge deflection, plays a key role in the outcome of the optimized nonlinear model due to the strong influence of the trailingedge deflection on the section lift coefficient. One of the main challenges of morphing aircraft is bridging the gap between two actuators without breaking the continuity of the surface while maintaining large strains. This model's section-based approach allows for easy incorporation of the SMTE's compliant sections in the trailing-edge shape function. The SMTE's six actuators move independently from one another yet remain horizontal while the flexible honeycomb with elastomeric skin linearly bridges the gap between the actuators. These transition sections are crucial to the continuous deformations of the SMTE; however, their inherent flexibility is unideal, and future designs can improve upon this aspect. A diagram of the modular structure of the SMTE can be seen in Fig. 4. The representative shape function describes the spanwise distribution in trailing-edge deflection for an arbitrary configuration and can be seen in Fig. 5. Mathematically, this is achieved by prescribing six inputs corresponding to the tip deflections of the six actuators on one wing and is represented as a constant value for the length of the actuator. Between actuators, the tip deflection is characterized as a linear function, as mentioned previously. Further details regarding the spanwise deformation in addition to the control derivatives of this morphing concept can be found in Pankonien et al. [22]. Last, the left and right wings are assumed to actuate symmetrically. From this, it is clear to imagine how more complex morphing shapes can be included in this model as morphing technologies progress, such as sinusoidal trailing-edge transitions, which are more representative of biological entities such as birds.

\section{Model Validation}

This model was validated against experimental data for a finite wing of NACA 0012 profile and aspect ratio 6 at $R e=1.5 \times 10^{5}$. The wing was constructed to scale for a small UAV with a chord of $0.305 \mathrm{~m}$ and was mounted vertically in the $1.5 \times 2.1 \mathrm{~m}$ closed-loop wind tunnel at the University of Michigan. This wing has the same geometric properties as the SMTE but is substantially more rigid, providing a more realistic comparison to the model presented, which does not include aeroelastic effects. Data were collected using a sixaxis force balance to obtain the $C_{L}-\alpha$ curves in addition to the drag

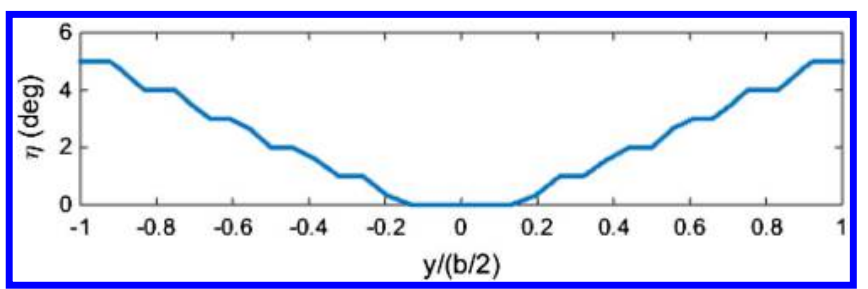

Fig. 5 Sample user-defined shape function representing the spanwise morphed deflection of the active and inactive sections, where $b$ represents the span.

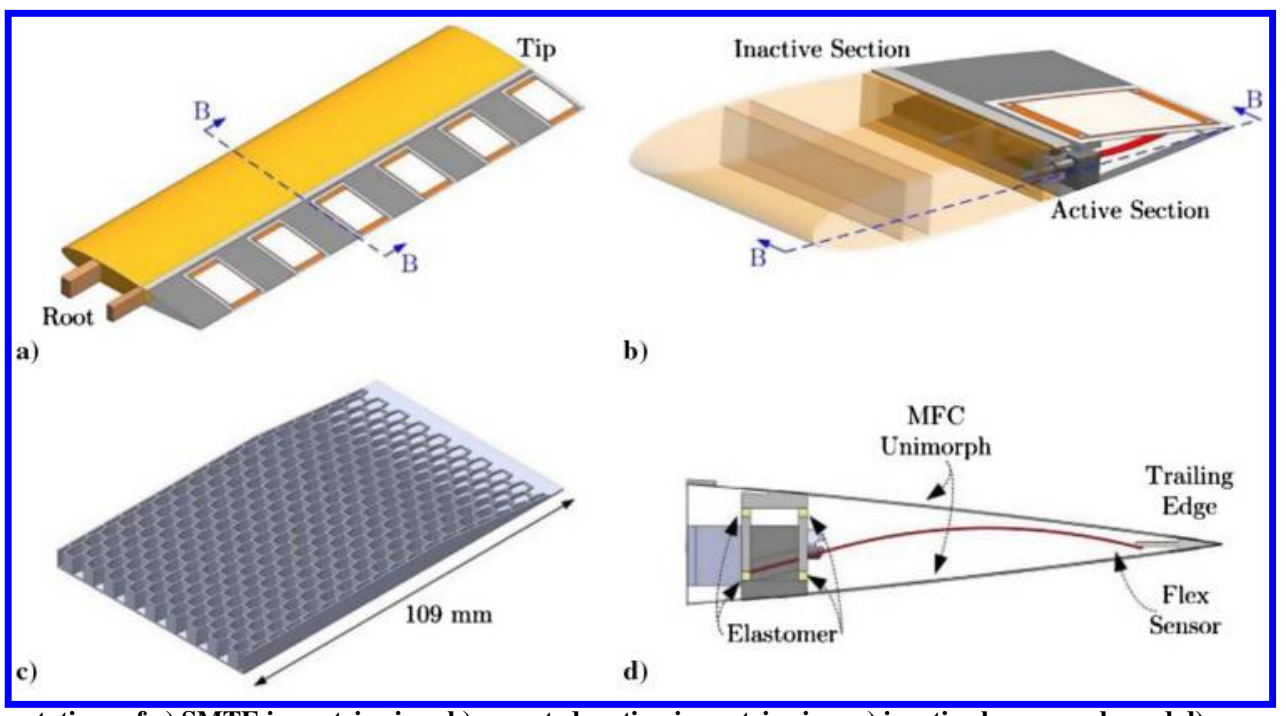

Fig. 4 Representations of a) SMTE isometric view, b) repeated section isometric view, c) inactive honeycomb, and d) cross-sectional view. 


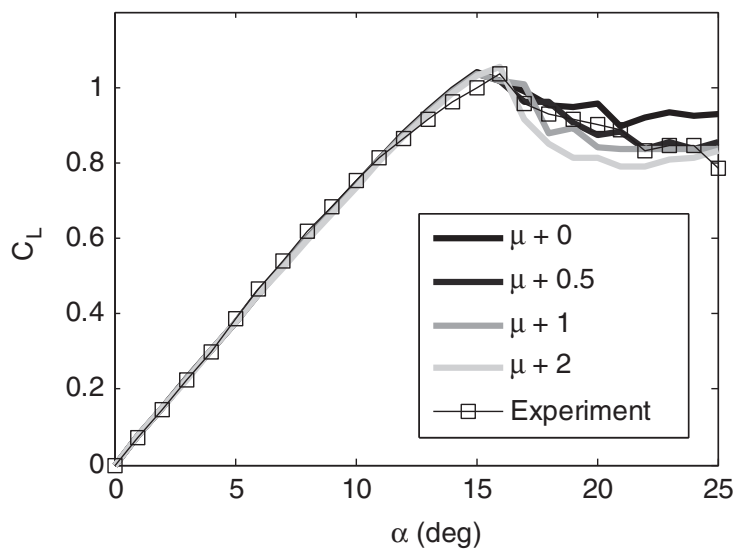

Fig. 6 Comparison of NACA 0012 experimental data to the nonlinear model with varying artificial viscosity [23].

and moment curves. Last, the system was automated using closedloop control to sweep through the angles of attack with accuracy up to $1 / 40$ of a degree. For further details of the experimental setup, see [15].

Shown in Fig. 6 is a comparison between the experimental results and the modeled prediction for zero actuation across the span of the wing. It is important to note that the artificial viscosity term is defined as a lower bound, meaning that the value of the artificial viscosity can be tuned if necessary. This is of importance for the cases tested here because the aerodynamic conditions are near turbulent transition, and thus discrepancies from the 2-D simulated data could misinform the model. Even poststall, the model is able to predict the nonlinear aerodynamic behavior granted with less accuracy than the linear region. These preliminary results indicate that an addition of 0.5 to the artificial viscosity term defined in Eq. (5) most accurately captures the experimental results and thus will be carried throughout the rest of this analysis. This factor can be further optimized and may vary when applied to other scenarios because it is dependent upon the relative accuracy between the poststall behavior of the 2-D simulation and the experimental data.

\section{E. Effect of Two-Dimensional Lift Curve}

As mentioned previously, the simulated 2-D curves play an important role in determining the results of the model and can thus provide insight into the limitations of the system even before optimization. This is evident when analyzing the effects of trailingedge displacement on the 2-D lift curves as seen in Fig. 7. Not only does deflecting the trailing edge upward, an expected response for stall recovery, generate negative lift which shifts the lift curve down, but it also shifts the curve to the right, thus delaying stall. However, the dominant response decreases the airfoil lift while the ability for the airfoil to delay stall is limited. This indicates that, for a given offdesign angle of attack, the airfoil may not physically be able to adapt to the on-design target lift condition. Therefore, the 2-D lift curves

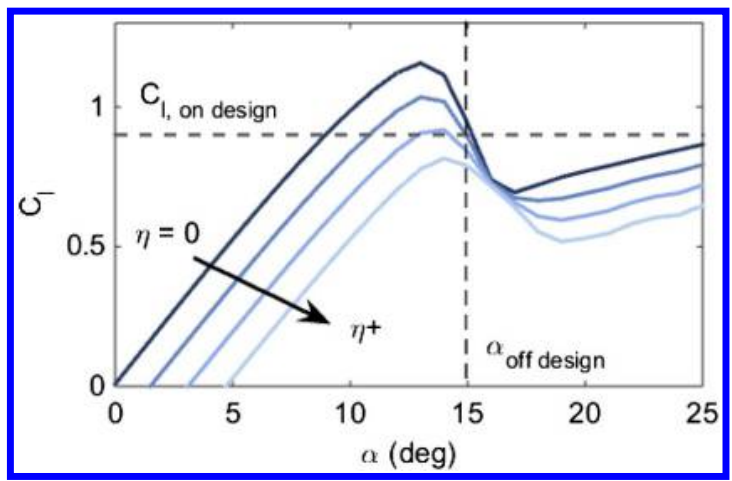

Fig. 7 Effect of the 2-D lift curve with respect to the trailing-edge deflection for an airfoil of geometry specified in Fig. 2. show that the ability of a morphing wing to recover from stall is dependent upon the magnitude of both the on-design lift condition and the off-design angle of attack. It follows that the problem under consideration may be bounded by combinations of the on- and offdesign conditions.

\section{Numerical Optimization}

Given that the wing under consideration presented in Fig. 4 has a total of six actuators, the most appropriate method of analyzing recovery via spanwise morphing is through optimization of the spanwise configuration using the nonlinear lifting-line model. This was achieved using a constrained minimization algorithm that tailored the actuator deflections, where now $\boldsymbol{\eta}$ is a vector, to minimize the total drag. The model was constrained to reach the on-design lift while maintaining the off-design angle of attack. Each actuator deflection was bounded by a maximum tip deflection of $8.85 \mathrm{deg}$ so as to not exceed the physical limitations of the wing, providing a realistic analysis for this particular morphing concept. Furthermore, because of the complex nature of optimizing a morphing wing, assuming an elliptical circulation distribution for the initial iteration does not always result in successful optimization. Instead, the converged circulation distribution for a wing with no spanwise variation in trailing-edge deflection was calculated at each angle of attack and was then used to seed the model.

For this optimization, there exists significant tradeoffs between the iterations to convergence within the desired error and the tolerance of the optimization. A strict convergence criteria of $0.01 \%$ error from the previous iteration was chosen for this model, defined by

$$
\text { \%error }=\left|\frac{\Gamma_{n-1}-\Gamma_{n}}{\Gamma_{n-1}}\right| * 100 \%
$$

Thus, circulations that did not meet this criterion for one or more spanwise sections were said to have not converged. Accordingly, by increasing the tolerance of the optimization function and lift constraint, the iterations required for the solution to converge increases. For practical purposes, the number of iterations was constrained to 2000 or less, limiting the tolerance to $1 \times 10^{-2}$ for the optimization function, output, and lift constraint. Given that, for Prandtl's finite wing theory [23], the slope of the linear region of the lift curve for a finite wing of aspect ratio 6 is expected to be $(2 \pi) * 0.75$, the error in angle of attack associated with a tolerance on $C_{L}$ of $1 \mathrm{e}-2$ is less than $0.1 \mathrm{deg}$. This tolerance was deemed to be adequate for this analysis.

\section{Results}

Using the methods described previously, the trailing-edge deflections of a finite wing were optimized to adapt to nonlinear aerodynamic conditions and return to the initial lift. The results of a single optimized case will be discussed before investigating the limitations of a morphing wing to adapt to stall conditions to demonstrate the effects of spanwise morphing on the aerodynamic behavior of the wing.

\section{A. Optimized Adaptation}

The wing was optimized to return to an on-design lift of $80 \%$ of $C_{L, \max }$ of the experimental data and an off-design angle of attack of $15 \mathrm{deg}$ to investigate the capabilities of the optimized nonlinear lifting-line model. This represents just one of multiple successful cases of adaptation, as will be discussed in the latter section. First, by generating the lift curve for the adapted configuration, the success of the optimization was determined. The goal was to achieve the target (on-design) lift for the given off-design angle of attack; however, recovery was only deemed successful when this occurred at an angle less than or equal to that associated with $C_{L, \max }$. As can be seen in Fig. 8, the case under investigation successfully recovered from stall because it matched the target lift for an angle of attack occurring prior for the adapted wing's stall angle. This can be further validated with the circulation distributions depicted in Fig. 9, which demonstrate 


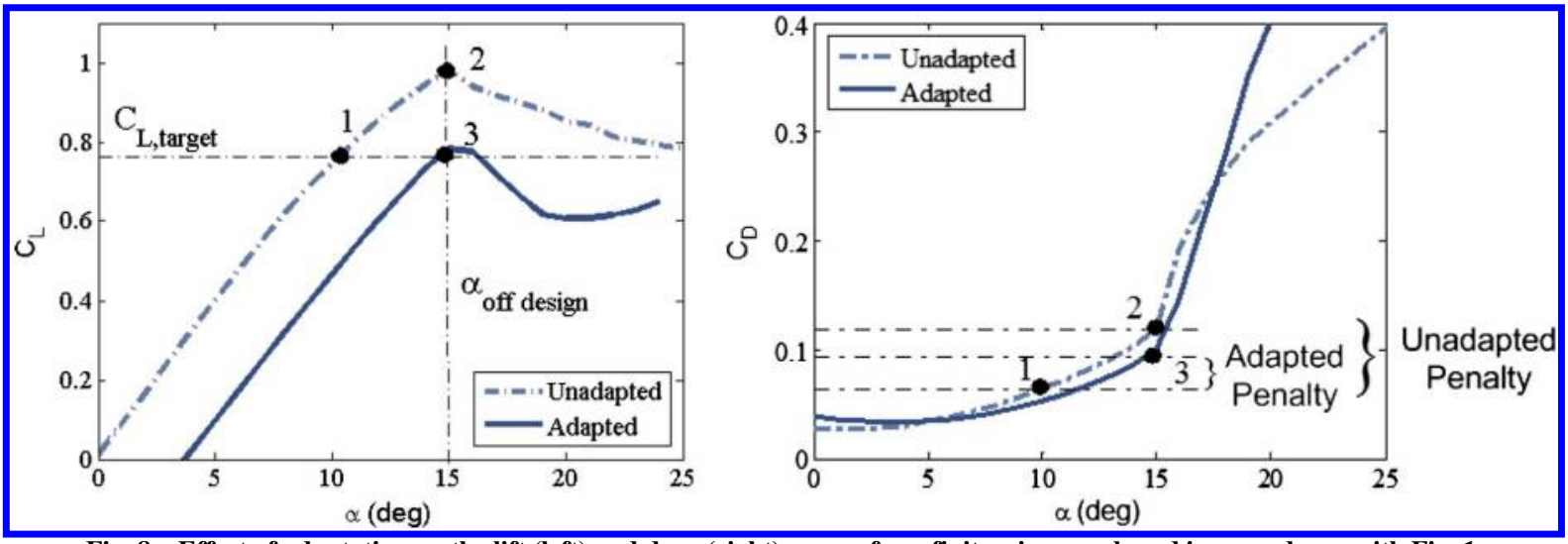

Fig. 8 Effect of adaptation on the lift (left) and drag (right) curves for a finite wing numbered in accordance with Fig. 1.

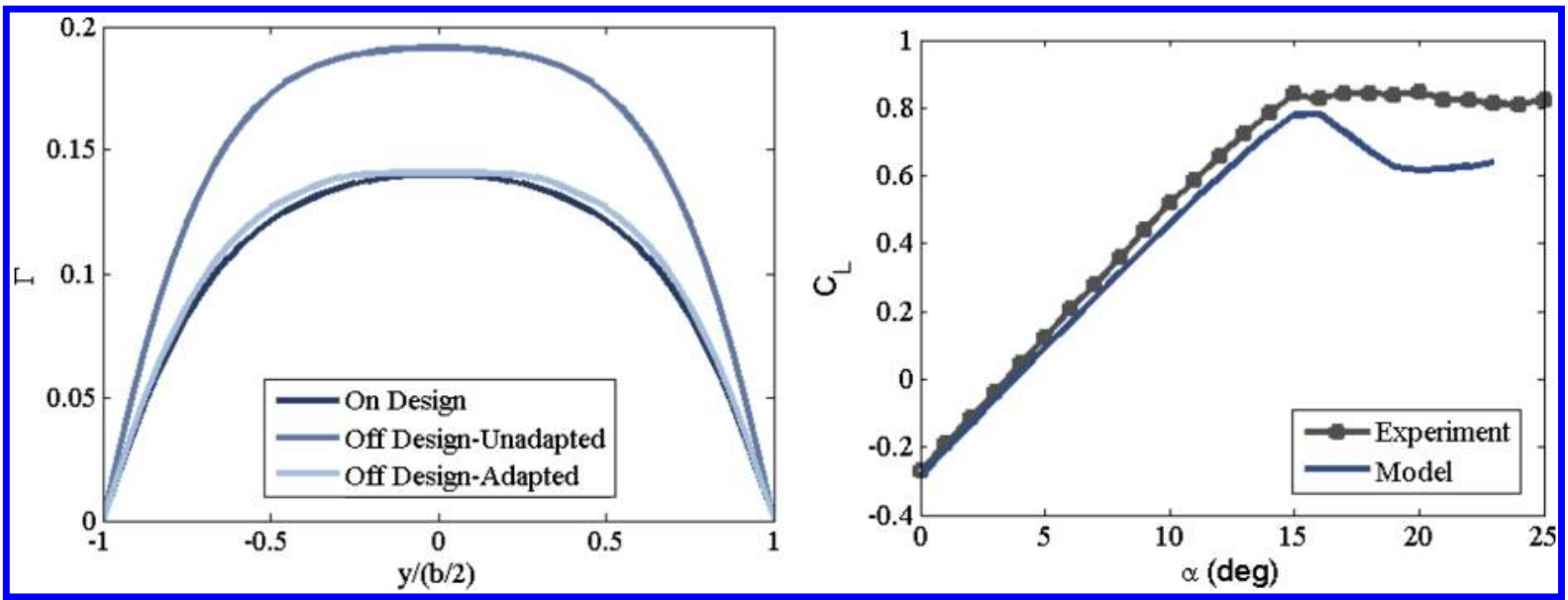

Fig. 9 Circulation distributions for $80 \% C_{L, \text { max }}$ at $15 \mathrm{deg}$ (left) and comparison of modeled results and experimental results of optimized configuration for $80 \% C_{L, \max }$ (right).

that the lift distribution is restored while in the off-design state. Recognizing that the on-design scenario represents the same flight condition as the reduced angle method, this demonstrates that tailoring the wing's geometry has the same effect as the traditional method of stall recovery.

Furthermore, the drag can be analyzed to quantify the performance improvement due to adaptation. The output of the optimization relies on a constrained minimization of the total drag and should thus optimize away from stall, where the drag increases dramatically. In the case presented here, the optimized configuration occurs just before the stall angle of the adapted lift curve, implying that the optimization may be near the limitations of the system. However, although the adapted configuration operates close to the stall angle, the drag penalties still indicated a significant improvement in the performance of the wing. The drag penalty for the unadapted configuration was 0.0545 , whereas the adapted configuration experienced a penalty of 0.0338 , resulting in a $38 \%$ reduction. This shows that, by morphing the wing to adapt, the drag penalty was significantly reduced. It is also worth noting that the reduction in drag penalty for successfully optimized cases will increase with off-design angle of attack. Because this particular case was analyzed near stall, this metric is a lower bound, and the performance benefits can potentially exceed this value substantially.

The results of the optimization were compared to experimental results conducted with the SMTE for proof of concept. The optimized actuator deflections for the case detailed previously were prescribed to the SMTE. The aerodynamic data were collected following the experimental setup and procedure described previously. A full description of the experimental setup can be found in [15]. As shown in Fig. 9, the model matches fairly well with the experimental data particularly at prestall angles but slightly underestimates the wing's lift overall. This can be attributed to differences between the underlying assumptions in the model and the physical attributes of the experimental wing. Specifically, this model does not include any effects of the compliance of the morphing wing trailing edge, particularly at the flexible transition areas, or aeroelastic effects of the wing in general, which would affect the overall aerodynamic behavior, especially poststall, due to passive deformations in the camber and twist of the wing. This could be improved upon with future iterations of the SMTE design in addition to incorporating aeroelastic effects into the model. Furthermore, the model does not fully capture the poststall behavior of the wing. This is due to the fact that the artificial viscosity term was tuned to match experimental data for a nonflexible wing that closely resembles the assumptions of the model. Because this method does not rely on the poststall behavior of the experimental results, this will not affect the model's ability to optimize the spanwise deflection to recover. Thus, the only discrepancies between the model and the experimental results are due to the compliance of the wing and do not affect the results of the model and the consequent analysis.

\section{B. Quantifying System Limitations}

By optimizing the spanwise trailing-edge deflections of a morphing wing for a single set of flight conditions, the wing successfully recovered from stall and returned to the on-design lift while maintaining the off-design angle of attack. This same procedure was conducted over a range of conditions to determine the limitations of a morphing wing such as the SMTE to adapt to both pre- and poststall conditions. For the unactuated geometry of the SMTE, the stall angle occurred at $15 \mathrm{deg}$ and will be useful when comparing the range of off-design cases to the initial configuration.

The results of the optimization, shown in Fig. 10, were broken down into four categories: recovered, unrecovered, possible solution, and no possible solution. Physically, a wing either can or cannot 
Possible Solution - Recovered - Unrecovered - No Solution

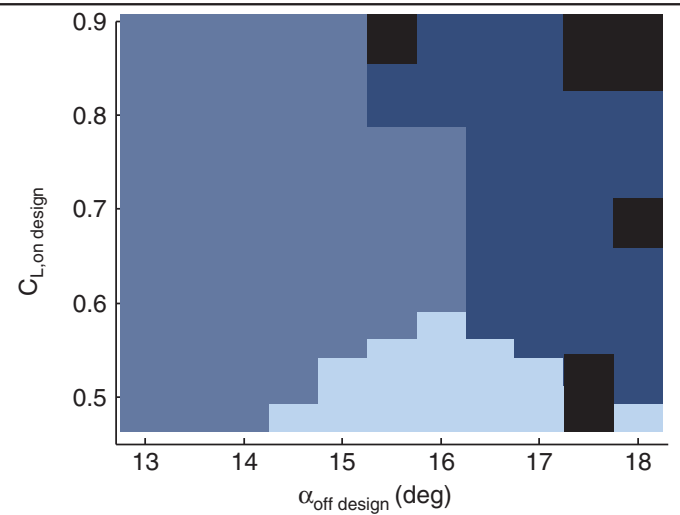

Fig. 10 Design space for a spanwise morphing wing with respect to adaptation.

recover from stall. A solution that recovered successfully adapted from the off-design condition such that the new flight condition occurred in a prestall region of the morphed wing's lift curve. This was assessed by comparing the off-design angle of attack to the angle of attack at maximum lift for the adapted lift curve. A solution that was unrecovered met the requirements defined in the optimization, yet the new flight condition occurred in the poststall region of the morphed wing's lift curve. Because this analysis includes a discussion on improving current morphing designs, a possible solution case was introduced upon observing that some results reached the maximum bounded value $(8.85 \mathrm{deg})$ for the tip deflection. This indicated that perhaps recovery for these cases would be possible with a different morphing mechanism if the bound on the tip deflection was larger. The final category, no possible solution, was added to accommodate cases where the optimization failed to find an appropriate solution. Although this case also represents a wing that has not recovered, it is distinguished from the unrecovered case in that the optimizer was not able to match the initial lift condition within the constraints and tolerances of the optimization. These cases are numerical artifacts, and some but not all can be resolved by decreasing the relaxation factor; however, increased underrelaxation further approximates the solution and substantially changes the poststall behavior.

The data show that the adaptability of a morphing wing to recover, especially from stall, is indeed limited. In fact, there appear to be three distinct regions that detail the conditions in which the wing is able to adapt. As expected, the wing was capable of adapting to off-design conditions that occur prestall; however, when the initial lift condition was low, the actuators could not provide sufficient deflection to match the on-design lift condition. This makes up the region of possible solutions. Furthermore, the wing was only able to adapt to off-design angles of attack that were at a maximum of $16 \mathrm{deg}, 1 \mathrm{deg}$ past the stall angle for the unactuated wing. This matches the 2-D trends noted previously in Fig. 7, where the 2-D lift curves indicated that deflecting the actuator up only slightly delayed stall by shifting the lift curve. Thus, for situations where stall recovery over a broad range of flight conditions is desirable, changes in the design of morphing mechanisms should be considered.

Given these results, the first proposed method of improving the adaptability of morphing structures is to increase the range of tip deflections. This addresses the region of possible solutions where the initial lift condition is low, correlating to a large change in lift between the on-design and off-design conditions. This limited range of actuation is a persistent issue in smart morphing materials, which do not rely on conventional actuators. One solution that retains the same morphing mechanisms assumed for this analysis would be to add an active anterior point of rotation, which can be actuated by another smart material like shape memory alloy (SMA) wires. Hence, the trailing-edge deflection would be compounded, including both the actuation of the SMA and MFC. The second improvement in morphing wings would require some mechanism of further delaying stall in the 2-D lift curves and preventing flow separation, while maintaining adequate lift. Coincidentally, the same hinge suggested previously could provide the solution. In actuating the SMA and MFC in opposite directions, the airfoil experiences reflex camber. Pankonien and Inman have shown that a morphing airfoil composed of an MFC trailing edge with anterior SMA hinge in reflex can achieve the same lift as an airfoil with monotonic actuation while maintaining attached flow [24]. Thus, a single improvement to existing morphing designs, a continuous morphing hinge in addition to the camber morphing control surface, is suggested for future morphing aircraft designs with an emphasis on broad-range stall recovery.

\section{Conclusions}

Morphing aircraft provide a unique ability to adapt to changes in flight conditions. In this work, a method was developed for analyzing the ability of morphing wings with arbitrary variations in spanwise camber to adapt to these changes. An extended nonlinear lifting-line model with low poststall fidelity was used to optimize the spanwise trailing-edge deflections of a morphing wing at the UAV scale with six camber-morphing actuators to adapt to nonlinear aerodynamics with the ability to recover from stall using nontraditional stall recovery methods. By tailoring the section-based approach of nonlinear lifting-line theory to morphing aircraft where the spanwise geometry varies, it was shown that a morphing wing can successfully adapt to nonlinear aerodynamics and reduce the drag penalty associated with operating at the off-design condition. Further investigation identified that the ability for this particular spanwise morphing wing to adapt to stall in particular was limited to midrange lift conditions relative to the maximum lift and angles of attack encompassing prestall and near stall conditions. These limitations can be overcome through the addition of a continuous morphing hinge, which would extend the range of actuation and allow for reflex camber. Thus, it is highly recommended that future designs within morphing aircraft encompass this additional degree of freedom, which allows for control over the compounded trailing-edge deflection and reflex camber of the airfoil.

\section{Acknowledgments}

This work is supported in part by the U.S. Air Force Office of Scientific Research under grant FA9550-16-1-0087, titled AvianInspired Multifunctional Morphing Vehicles, monitored by B. L. Lee.

\section{References}

[1] Lentink, D., Müller, U. K., Stamhuis, E. J., De Kat, R., Van Gestel, W., Veldhuis, L. L. M., Henningsson, P., Hedenström, A., Videler, J. J., and Van Leeuwen, J. L., "How Swifts Control Their Glide Performance with Morphing Wings," Nature, Vol. 446, No. 7139, 2007, pp. 1082-1085. doi:10.1038/nature05733

[2] Gillies, J. A., Thomas, A. L. R., and Taylor, G. K., "Soaring and Manoeuvring Flight of a Steppe Eagle Aquila Nipalensis," Journal of Avian Biology, Vol. 42, No. 5, 2011, pp. 377-386. doi:10.1111/j.1600-048X.2011.05105.x

-[3] Barbarino, S., Bilgen, O., Ajaj, R. M., Friswell, M. I., and Inman, D. J., "A Review of Morphing Aircraft," Journal of Intelligent Material Systems and Structures, Vol. 22, No. 9, 2011, pp. 823-877. doi:10.1177/1045389X11414084

[4] Urnes, J., Nguyen, N. T., and Dykman, J., "Development of Variable Camber Continuous Trailing Edge Flap System," Proceedings of the Fundamental Aeronautics Technical Conference, NASA Paper 15ATC-0250, Cleveland, OH, March 2012.

[5] Kaul, U. K., and Nguyen, N. T., "Drag Optimization Study of Variable Camber Continuous Trailing Edge Flap (VCCTEF) Using OVERFLOW," 32nd AIAA Applied Aerodynamics Conference, AIAA Paper 2014-2444, 2014.

[6] Kaul, U. K., and Nguyen, N. T., "A 3-D Computational Study of a Variable Camber Continuous Trailing Edge Flap (VCCTEF) Spanwise Segment," 33rd AIAA Applied Aerodynamics Conference, AIAA Paper 2015-2422, June 2015. 
[7] Kota, S., Hetrick, J. A., Osborn, R., Paul, D., Pendleton, E., Flick, P., and Tilmann, C., "Design and Application of Compliant Mechanisms for Morphing Aircraft Structures," Proceedings of SPIE, Smart Structures and Materials, Vol. 5054, San Diego, CA, Aug. 2003, pp. 24-33. doi: $10.1117 / 12.483869$

[8] Bilgen, O., Kochersberger, K. B., and Inman, D. J., "Novel, Bidirectional, Variable-Camber Airfoil via Macro-Fiber Composite Actuators," Journal of Aircraft, Vol. 47, No. 1, 2010, pp. 303-314.

[9] Pankonien, A. M., and Inman, D. J., "Experimental Testing of SpanWise Morphing Trailing Edge Concept," Proceedings of SPIE, Active and Passive Smart Structures and Integrated Systems, Vol. 8688, April 2013. doi: $10.1117 / 12.2009400$

[10] Pankonien, A. M., and Inman, D. J., "Aerodynamic Performance of a Spanwise Morphing Trailing Edge Concept," Proceedings of the 25th International Conference on Adaptive Structures and Technologies, The Hague, The Netherlands, 2014, Paper 004.

[11] Pankonien, A. M., and Inman, D. J., "Spanwise Morphing Trailing Edge on a Finite Wing," Proceedings of SPIE, Active and Passive Smart Structures and Integrated Systems, Vol. 9431, April 2015. doi: $10.1117 / 12.2083945$

[12] Sivells, J. C., and Neely, R. H., "Method for Calculating Wing Characteristics by Lifting-Line Theory Using Nonlinear Section Lift Data," NASA Rept. 865, 1947.

[13] Piszkin, S. T., and Levinsky, E. S., "Nonlinear Lifting Line Theory for Predicting Stalling Instabilities on Wings of Moderate Aspect Ratio," General Dynamics Rept. ADA027645, San Diego, CA, June 1976.

[14] Chattot, J. J., "Analysis and Design of Wings and Wing/Winglet Combinations at Low Speeds," 42nd AIAA Aerospace Science Meeting and Exhibit, AIAA Paper 2004-0220, Jan. 2004.

[15] Gamble, L. L., Pankonien, A. M., and Inman, D. J., "Stall Recovery of the Span-Wise Morphing Trailing Edge Concept via an Optimized Nonlinear Model," Proceedings of the 26th International Conference on Adaptive Structures and Technologies, Curran Associates, Inc., New York, 2016, pp. 36-49.
[16] Aranake, A. C., Lakshminarayan, V. K., and Duraisamy, K. "Computational Analysis of Shrouded Wind Turbine Configurations Using a 3-Dimensional RANS Solver," Renewable Energy, Vol. 75, March 2015, pp. 818-832. doi:10.1016/j.renene.2014.10.049

-[17] Bremseth, J., and Duraisamy, K., "Theoretical and Computational Analysis of Vertical Axis Wind Turbine Arrays," Theoretical and Computational Fluid Dynamics, Vol. 30, No. 5, 2016, pp. 387-401.

[18] Pankonien, A. M., "Smart Material Wing Morphing for Unmanned Aerial Vehicles," Ph.D. Dissertation, Aerospace Dept., Univ. of Michigan, Ann Arbor, MI, 2015.

[19] Chattot, J. J., "Low Speed Design and Analysis of Wing/Winglet Combinations Including Viscous Effects," Journal of Aircraft, Vol. 43, No. 2, 2006, pp. 386-389. doi:10.2514/1.15349

[20] Chattot, J. J., "Optimization of Wind Turbines Using Helicoidal Vortex Model," Journal of Solar Energy Engineering, Vol. 125, No. 4, 2003, pp. 418-424. doi: $10.1115 / 1.1621675$

[21] Gallay, S., Ghasemi, S., and Laurendeau, E., "Sweep Effects on NonLinear Lifting Line Theory near Stall," 52nd Aerospace Science Meeting, AIAA Paper 2014-1105, 2014.

[22] Pankonien, A. M., Gamble, L. L., and Inman, D. J., "Aerodynamic Performance of the Spanwise Morphing Trailing Edge Concept," Journal of Intelligent Material Systems and Structures (in review).

[23] Anderson, J. D., Fundamentals of Aerodynamics, 1st ed., McGraw-Hill, New York, 1984, Chap. 5.

[24] Pankonien, A. M., and Inman, D. J., "Synergistic Smart Morphing Aileron: Experimental Quasi-Static Performance," Journal of Intelligent Material Systems and Structures, Vol. 26, No. 10, 2015, pp. 1179-1190. doi: $10.1177 / 1045389 \times 14538530$

M. M. Choudhari Associate Editor 
This article has been cited by:

1. L. L. Gamble, D. J. Inman. 2017. Why morphology matters in birds and UAV's: How scale affects attitude wind sensitivity. Applied Physics Letters 111:20, 203701. [Crossref] 